\title{
A Low Complexity STPAP Algorithm based on Alternating Polarization Sensitive Array
}

\section{Shuang Sha ( $\nabla$ whsha_1994@163.com )}

Ocean University of China - Laoshan Campus: Ocean University of China https://orcid.org/0000-00018719-0158

\section{Tingting Lyu}

Ocean University of China - Laoshan Campus: Ocean University of China

Hao Zhang

Ocean University of China - Laoshan Campus: Ocean University of China

\section{T. Aaron Gulliver}

University of Victoria

\section{Research}

Keywords: Alternate Polarization Sensitive Array, Polarization Sensitive Array, Space-Time-Polarization Adaptive Processing, LCMV

Posted Date: April 19th, 2021

DOl: https://doi.org/10.21203/rs.3.rs-422916/v1

License: (c) (i) This work is licensed under a Creative Commons Attribution 4.0 International License. Read Full License

Version of Record: A version of this preprint was published at EURASIP Journal on Advances in Signal Processing on March 21st, 2022. See the published version at https://doi.org/10.1186/s13634-02200859-2. 


\title{
A low complexity STPAP algorithm based on alternating polarization sensitive array
}

\author{
Shuang Sha ${ }^{1}$, Tingting Lyu ${ }^{1 *}$, Hao Zhang ${ }^{1,2}$, T. Aaron Gulliver ${ }^{2}$ \\ ${ }^{1}$ Institution of Information Science and Engineering, Ocean University of China, Qingdao, 266100, China \\ Open Studio for Marine High Frequency Communications, Pilot National Laboratory for Marine Science and \\ Technology (Qingdao). \\ ${ }^{2}$ Dept. of electrical and computer engineering, University of Victoria, Victoria, V8P 5C2, Canada \\ *tingtinglu@ ouc.edu.cn
}

\begin{abstract}
Background: Space-time adaptive processing (STAP) has been widely used in the fields of communication, radar, and navigation anti-jamming. However, the traditional scalar array used by STAP has certain limitations, because it can only obtain spatial information. In order to further improve the performance of the space-time domain joint filtering technology, this paper replaces the traditional scalar array with an alternating polarization sensitive array (APSA). Compared with the dual polarization sensitive array (DPSA), it can not only obtain the polarization information of the signal, but also reduce the computational complexity of the algorithm.
\end{abstract}

Methods: Using the polarization information of the signals, this paper proposes an alternate polarization sensitive array spacetime-polarization adaptive processing algorithm (APSA-STPAP) based on the linear variance minimum criterion (LCMV). Different from the traditional LCMV criterion, this paper takes the space-time polarization joint steering vector of the desired signal and the interference signal as the constraint matrix, and uses the " 1 condition" and "zero condition" as the constraint conditions to effectively suppress the interference signal and enhance the expectation signal.

Results: The simulation results show that: (1) APSA-STPAP algorithm can achieve the same filtering effect as DPSA-STPAP algorithm. From the perspective of the spatial domain, time domain and polarization domain, it can form null in the direction of interference, effectively suppress the interference signal, and realize space-time-polarization adaptive processing. (2) Under the same conditions, APSA-STPAP algorithm can achieve the same filtering effect as DPSA-STPAP algorithm. there is a big difference between the two algorithms, APSA-STPAP algorithm can effectively reduce the amount of computation. Moreover, the dipole of alternating polarization sensitive array is halved, which reduces the coupling effect between electric dipoles, and is conducive to engineering implementation. (3) APSA-STPAP algorithm can maintain good anti-interference performance even when the electric dipole and anti-jamming degree of freedom are reduced by half, and its anti-jamming performance is similar to that of polarization sensitive array. The output SINR of DPSA-STPAP algorithm is about 3dB higher than that of APSASTPAP algorithm. There is little difference between the anti- interference performance of APSA and DPSA.

\section{Key words: Alternate Polarization Sensitive Array; Polarization Sensitive Array; Space-Time-Polarization Adaptive Processing; LCMV}

\section{Introduction}

Antenna array processing techniques with spatial filtering have been shown to be effective in cancelling interference for the communication filed, especially in GNSS and radar ${ }^{[1]}$. With this approach, weights are applied to the signals received by different array elements to form nulls in the directions of the interference while steering the array response towards the desired signals. The weights of the array elements can be changed adaptively according to the signal environment ${ }^{[2]}$. Space-time adaptive processing (STAP) is based on spatial filtering ${ }^{[3]}$. In this case, each array element is followed by a finite impulse response (FIR) filter for timedomain filtering. STAP has been used to adaptively process radar signals using space-time two-dimensional sampling ${ }^{[4][5]}$. This solves the problem of ground clutter suppression in radar signals. STAP has also been applied for anti-interference in

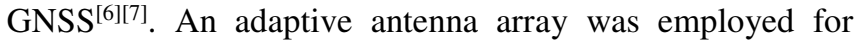
interference reduction which increases the number of degrees the freedom for signal processing. However, if the interference directions are close to those of the desired signals,
STAP will also degrade the desired signals when nulling the interference. Thus, polarization domain information is utilized to distinguish and suppress the interference. In [7][12], space-polarization adaptive processing (SPAP) was introduced to suppress interference in the joint spacepolarization domain. Furthermore, STPAP was proposed in [13] to mitigate interference. The polarization information of a signal can be obtained using a dual polarization sensitive array (DPSA). Compared with traditional antenna arrays, DPSA has many advantages such as strong interference suppression, robust detection, higher resolution, and polarization multiple access ${ }^{[14][17]}$. As a consequence, DPSA has been widely employed in radar, remote sensing, seismic signal processing, and wireless communication systems ${ }^{[16]}$. However, the complex structure and implementation difficulties limit DPSA use. Thus, an alternate polarization sensitive array (APSA) was proposed as an alternative ${ }^{[18]}$. A DPSA is composed of electric dipoles along the $\mathrm{x}$ and $\mathrm{y}$ axes which can receive electric field information from the $\mathrm{x}$ and $\mathrm{y}$ directions [18][20]. The APSA structural complexity is half that of DPSA, but the polarization information is also reduced 
by half compared to a PSA with the same array elements. However, their filtering performance is similar ${ }^{[20]}$.

In this paper, APSA is applied to space-timepolarization adaptive processing, and an alternative polarization sensitive array space time polarization adaptive processing (APSA-STPAP) algorithm based on LCMV criterion is formed. Based on the traditional LCMV criterion, the joint steering loss of the desired signals and the interference signals in the spatial domain, time domain and polarization domain are taken as the constraint matrix, and the "set 1 " and "set 0 " conditions are taken as the constraint conditions to effectively suppress the interference signals and enhance the desired signals.

The remainder of this paper is organized as follows. Section 2 presents the polarization concept, the APSA structure, and the signal model. The proposed APSA-STPAP filtering algorithm based on the LCMV criterion is given in Section 3. Simulation results are presented in Section 4 to evaluate the performance and effectiveness of the proposed algorithm. Moreover, the proposed APSA-STPAP algorithm is compared with the DPSA-STPAP algorithm. Finally, Section 5 concludes this paper.

\section{Alternating polarization sensitive array and the received signal model}

\subsection{Polarization model}

Figure 1 illustrates a transverse electric (TE) wave incident from the direction $(\theta, \varphi)$ with respect to the reference point $\mathrm{O}$, where $\theta \in\left[-90^{\circ}, 90^{\circ}\right]$ is the pitch angle and $\varphi \in\left[0^{\circ}, 360^{\circ}\right]$ is the azimuth angle ${ }^{[21]}$. The pitch angle refers to the acute angle between the direction of the incoming signal and the normal of the antenna. The azimuth angle refers to the angle between the projection of the incoming signal on the antenna and the reference direction. Denote the transient electric field vector at point $\mathrm{P}$ as $\vec{E}$ which can be written as $\vec{E}=E_{\theta} \vec{e}_{\theta}+E_{\varphi} \vec{e}_{\varphi}$, where $\left(\vec{e}_{\theta}, \vec{e}_{\varphi}\right)$ represents a pair of orthonormal vector and $E_{\theta}$ and $E_{\varphi}$ denote the transient projections in the $\vec{e}_{\theta}$ and $\vec{e}_{\varphi}$ directions, respectively. The magnitudes of the electric field components are $E_{\theta}=\sin \gamma \mathrm{e}^{j \eta}$ and $E_{\varphi}=\cos \gamma$ where $(\gamma, \eta)$ represents the polarization parameters which describe the polarization mode of the TE wave. $\gamma \in\left[0^{\circ}, 90^{\circ}\right]$ represents the amplitude ratio between the horizontal and vertical components of the electric field and $\eta \in\left[-180^{\circ}, 180^{\circ}\right]$ denotes the phase difference between the horizontal and vertical components of the electric field. According to the orientation of the endpoint of the transient electric field vector, the TE wave can be classified as linear polarization, circular polarization, or elliptical polarization $(\mathrm{EP})^{[22]}$. Moreover, linear polarization can be classified as horizontal polarization (HP) or vertical polarization (VP). Circular polarization can be classified as right-handed circular polarization (RHCP) or left-handed circular polarization (LHCP). These polarization modes can be defined by the polarized parameters given in Table 1 . The polarization vector of the received signal at the array can be expressed as ${ }^{[23][24]}$.

$$
\mathbf{S}_{p}=\left[\begin{array}{l}
E_{x} \\
E_{y} \\
E_{z} \\
H_{x} \\
H_{y} \\
H_{z}
\end{array}\right]=\left[\begin{array}{lr}
-\sin \varphi & \cos \theta \cos \varphi \\
\cos \varphi & \cos \theta \sin \varphi \\
0 & -\sin \theta \\
\cos \theta \cos \varphi & \sin \varphi \\
\cos \theta \sin \varphi & -\cos \varphi \\
-\sin \theta & 0
\end{array}\right]\left[\begin{array}{l}
\cos \gamma \\
\sin \gamma e^{j \eta}
\end{array}\right](1)
$$

\subsection{Alternating polarization sensitive array structure}

A PSA contains M dual-polarized elements as shown in Figure 2. The polarization information is obtained through correlation of the $\mathrm{x}$ and $\mathrm{y}$ channels and the spatial information is obtained from the phase lag. Although the array can have any shape, a uniform linear array (ULA) along the y-axis is considered here with azimuth angle $\varphi=90^{\circ}$. The distance between two elements, $d$, is assumed to be half the wavelength of a desired signal $(d=\lambda / 2)$.

The APSA used here is based on a DPSA and is shown in Figure 3 [18][20]. Compared to a DPSA, each array element is along the $\mathrm{x}$-axis or $\mathrm{y}$-axis direction of the dipole and they alternate.

Fig. 4 illustrates the structure of the alternating polarization sensitive array space-time-polarization adaptive processing (APSA-STPAP) algorithm. This is similar to a conventional STAP with the LCMV algorithm which has one $\mathrm{RF}$ chain for each antenna element ${ }^{[25]}$, but here there are RF chains from the vertical or horizontal dipole components. After the RF front end, signals from each antenna component pass through a finite impulse response (FIR) filter with $\mathrm{K}$ taps.

\subsection{Array Steering Vector}

The joint space time polarization steering vector for a dual-polarized ULA array $s_{S T P}$ can be expressed as

$$
s_{S T P}(\theta, f, \gamma, \eta)=s_{S}(\theta) \otimes s_{P}(\gamma, \eta) \otimes s_{T}(f)
$$

where $\otimes$ denotes the Kronecker product. The length space steering vector is

$$
\begin{aligned}
\mathbf{s}_{S}(\theta) & =\left[\begin{array}{llll}
1 q^{1} & q^{2} \cdots & q^{M-1}
\end{array}\right]^{T} \\
& =\left[1 \exp \left(-j 2 \pi \frac{d}{\lambda} \sin \theta\right) \cdots \exp \left(-j 2 \pi(M-1) \frac{d}{\lambda} \sin \theta\right)\right]^{T}
\end{aligned}
$$

where $q=e^{-j 2 \pi \frac{d}{\lambda} \sin \theta}$ is the spatial phase factor. The length $K$ time-domain steering vector can be expressed as

$$
\mathbf{s}_{T}(f)=\left[1 \exp \left(-j 2 \pi f T_{s}\right) \cdots \exp \left(-j 2 \pi(K-1) f T_{s}\right)\right]^{T}
$$

where $f$ is the intermediate frequency after the signal passes through the RF front end and $T_{s}$ is the sampling period of the FIR filter.

From (1), The polarization-domain steering vector for a dual-polarized ULA array can be written as 


$$
\mathbf{s}_{P}(\theta, \quad \gamma, \quad \eta)=\left[\begin{array}{cc}
-1 & 0 \\
0 & \cos \theta
\end{array}\right]\left[\begin{array}{l}
\cos \gamma \\
\sin \gamma e^{j \eta}
\end{array}\right]=\left[\begin{array}{l}
-\cos \gamma \\
\cos \theta \sin \gamma e^{j \eta}
\end{array}\right]
$$

Similar to (2), the joint polarization space-time steering vector for an alternating polarization sensitive array $S_{\text {ASTP }}$ is given by

$$
s_{A S T P}(\theta, f, \gamma, \eta)=s_{A S}(\theta) \otimes s_{A P}(\gamma, \eta) \otimes s_{T}(f)
$$

The length $\frac{M}{2}$ space steering vector for an APSA can be written as

$$
\begin{aligned}
\mathbf{s}_{S}(\theta) & =\left[1 q^{2} q^{4} \cdots q^{M-2}\right]^{T} \\
& =\left[1 \exp \left(-j 4 \pi \frac{d}{\lambda} \sin \theta\right) \cdots \exp \left(-j 2 \pi(M-2) \frac{d}{\lambda} \sin \theta\right)\right]^{T}
\end{aligned}
$$

The distance between adjacent dipoles, $\mathrm{d}$, is assumed to be the wavelength of a signal. The polarization-domain steering vector for an APSA can be expressed as

$$
\begin{aligned}
s_{P}(\theta, \quad \gamma, \quad \eta) & =\left[\begin{array}{cc}
-1 & 0 \\
0 & \cos \theta
\end{array}\right]\left[\begin{array}{l}
\cos \gamma \\
q \sin \gamma e^{j \eta}
\end{array}\right] \\
& =\left[\begin{array}{l}
-\cos \gamma \\
\cos \theta \sin \gamma e^{j\left(\eta+2 \pi \frac{d}{\lambda} \sin \theta\right)}
\end{array}\right]
\end{aligned}
$$

Comparing (2) and (6), the dimension of the APSA joint steering vector is half that of DPSA. This lowers the number of calculations required and this also the computational complexity.

\subsection{Array Signal Receiving Model} is given by

The nth, $1 \leq n \leq L$, signal block received by the array

$$
\mathbf{X}(n)=\mathbf{A s}(n)+\mathbf{J}(n)+\mathbf{V}(n)
$$

where $\mathbf{A}$ is the joint vector matrix, $\mathbf{s}(n)$ is the amplitude vector of the desired signal, $\mathbf{J}(n)$ is the interference signal matrix, and $\mathbf{V}(n)$ is a white Gaussian noise vector with elements having mean 0 and variance $\sigma^{2}$. This can be expressed as

$$
\mathbf{X}(n)=\left[x_{11}(n), \cdots, x_{1 K}(n), \cdots, x_{M 1}(n), \cdots, x_{M K}(n)\right]^{T}
$$

Assume the pth, $p=1,2, \cdots, P$, signal has intermediate frequency $f_{p}$, incident angle $\theta_{p}$, and polarization parameters $\left(\gamma_{p}, \eta_{p}\right)$, and the qth, $q=1,2, \cdots, Q$, interference signal has intermediate frequency $f_{q}$, incident angle $\theta_{q}$, and polarization parameters $\left(\gamma_{q}, \eta_{q}\right)$. Then from Figure 4, the received signal model for the nth block can be written as

$$
\begin{aligned}
x_{A S T P}(n) & =\sum_{p=1}^{P} \mathbf{s}_{A S T P}\left(\theta_{p}, f_{p}, \gamma_{p}, \eta_{p}\right) s_{p}(n) \\
& +\sum_{q=1}^{Q} \mathbf{s}_{A S T P}\left(\theta_{q}, f_{q}, \gamma_{q}, \eta_{q}\right) j_{q}(n)+v_{A S T P}(n)
\end{aligned}
$$

\section{APSA-STPAP algorithm based on LCMV}

Assuming that the direction of arrival (DOA) and the polarization information of the desired signal is known, the LCMV criterion can be employed in the APSA-STPAP algorithm. The array output can be expressed as [26].

$$
y(n)=\mathbf{w}^{H} x_{A S T P}(n)
$$

where w is a length $M \times K$ weight vector and $(\cdot)^{H}$ denotes Hermitian. This vector can be written as

$$
\mathbf{w}=\left[w_{11}, \cdots, w_{1 K}, \cdots, w_{M 1}, \cdots, w_{M K}\right]
$$

The output signal power is

$$
\begin{aligned}
P & =E\left[|y(n)|^{2}\right] \\
& =E\left[\mathbf{w}^{H} x_{A S T P}(n) x_{A S T P}^{H}(n) \mathbf{w}\right] \\
& =\mathbf{w}^{H} \mathbf{R} \mathbf{w}
\end{aligned}
$$

where $\mathbf{R}=E\left[x_{A S T P}(n) x_{A S T P}^{H}(n)\right]$ is the covariance matrix of the received signals and noise. In practice, this matrix is unknown but the covariance matrix of the received signal obtained from a sampled covariance matrix [14] can be obtained as

$$
\hat{\mathbf{R}}=\frac{1}{L} \sum_{l=1}^{L} x_{A S T P}(n) x_{A S T P}^{H}(n)
$$

where $\mathrm{L}$ is the number of samples.

In order to ensure that the desired signals from $\theta_{p}$ is received correctly and the interference from $\theta_{q}$ is completely suppressed, the constraint condition of the weight vector can be expressed as follows:

$$
\left\{\begin{array}{l}
\mathbf{w}^{H} \mathbf{s}_{\text {ASTP }}\left(\theta_{p}, f_{p}, \gamma_{p}, \eta_{p}\right)=1 \\
\mathbf{w}^{H} \mathbf{s}_{\text {ASTP }}\left(\theta_{q}, f_{q}, \gamma_{q}, \eta_{q}\right)=0
\end{array}\right.
$$

The constraint condition in the direction of the desired signals is "set to 1", and the constraint condition in the direction of the interference is "set to 0". And because it forces the direction of the beam pattern of the receiving array to point to the desired direction, and the "zero point" to point to the interference direction.

To ensure signal reception in the directions of the $\mathrm{P}$ desired signals and the null to suppress interference in the direction of the $\mathrm{Q}$ interfering signals, the constraints on the weight vector can be expressed as

$$
\mathbf{C}^{H} \mathbf{w}=\mathbf{f}
$$

where $\mathbf{C}$ is an $M K \times(P+Q)$ constraint matrix and $\mathbf{f}$ is the response vector. They can be written as

$$
\begin{gathered}
\mathbf{C}=\left[\mathbf{s}_{A S T P}\left(\theta_{1}, f_{1}, \gamma_{1}, \eta_{1}\right), \cdots, \mathbf{s}_{A S T P}\left(\theta_{P}, f_{P}, \gamma_{P}, \eta_{P}\right),\right. \\
\left.\mathbf{s}_{A S T P}\left(\theta_{P+1}, f_{P+1}, \gamma_{P+1}, \eta_{p+1}\right), \cdots \mathbf{s}_{A S T P}\left(\theta_{P+Q}, f_{P+Q}, \gamma_{P+Q}, \eta_{p+Q}\right)\right] \\
\mathbf{f}=\underbrace{[1, \cdots, \underbrace{1,0 \cdots 0]^{T}}_{Q}}_{P}
\end{gathered}
$$

The optimization problem can be expressed as

$$
\begin{cases}\min & \mathbf{w}^{H} \hat{\mathbf{R}} \mathbf{w} \\ \text { s.t. } & \mathbf{C}^{H} \mathbf{w}=\mathbf{f}\end{cases}
$$

Using Lagrange multipliers, the optimal LCMV weight vector is ${ }^{[26]}$

$$
\mathbf{W}=\hat{\mathbf{R}}^{-1} \mathbf{C}\left(\mathbf{C}^{H} \hat{\mathbf{R}}^{-1} \mathbf{C}\right)^{-1} \mathbf{f}
$$


Array pattern is the response to different angle signals, is shown that

$$
F(\theta, f, \gamma, \eta)=\mathbf{w}^{H} \mathbf{s}_{A S T P}(\theta, f, \gamma, \eta)
$$

The array pattern normalized by absolute value is as follows:

$$
G(\theta, \varphi, f, \gamma, \eta)=20 \log _{10} \frac{|F(\theta, \varphi, f, \gamma, \eta)|}{|F(\theta, \varphi, f, \gamma, \eta)|}
$$

The signal covariance matrix $\mathbf{R}_{s}$ and interference plus noise covariance matrix $\mathbf{R}_{j+v}$ are

$$
\begin{gathered}
\mathbf{R}_{S}=E\left[x_{s}(n) x_{s}^{H}(n)\right] \\
\mathbf{R}_{j+v}=E\left[x_{j+v}(n) x_{j+v}^{H}(n)\right]
\end{gathered}
$$

Then the signal power at the output after beamforming can be written as

$$
\begin{aligned}
P_{s} & =E\left[\left|y_{s}(n)\right|^{2}\right] \\
& =E\left[\mathbf{w}^{H} x_{s}(n) x_{s}^{H}(n) \mathbf{w}\right] \\
& =\mathbf{w}^{H} \mathbf{R}_{s} \mathbf{w}
\end{aligned}
$$

and the interference plus noise power at the output as

$$
\begin{aligned}
& P_{j+v}=E\left[\left|y_{j+v}(n)\right|^{2}\right] \\
& =E\left[\mathbf{w}^{H} x_{j+v}(n) x_{j+v}^{H}(n) \mathbf{w}\right] \\
& =\mathbf{w}^{H} \mathbf{R}_{j+v} \mathbf{W}
\end{aligned}
$$

The signal to interference plus noise ratio (SINR) is defined as the ratio of signal power to interference plus noise power at the output

$$
\operatorname{SINR}=\frac{P_{s}}{P_{j+v}}=\frac{\mathbf{w}^{H} \mathbf{R}_{s} \mathbf{w}}{\mathbf{w}^{H} \mathbf{R}_{j+v} \mathbf{w}}
$$

\section{Simulation results}

\subsection{Array orientation diagram Comparison between APSA-STPAP algorithm and DPSA- STPAP algorithm}

An eight-element linear uniform APSA with one wavelength spacing (in each direction) is used. Each APSA dipole is followed by a tapped delay line with four taps. The desired signal is a bandwidth of $20.46 \mathrm{MHz}$ and an intermediate frequency of $46.52 \mathrm{MHz}$. The sampling frequency and number of samples are $62 \mathrm{MHz}$ and 300 , respectively. The signal to noise ratio (SNR) of the desired signal is $-20 \mathrm{~dB}$, and the interference to noise ratio (INR) of the interference signals is $50 \mathrm{~dB}$. Additive white Gaussian noise (AWGN) is assumed with mean 1 and variance 0 . Since the joint polarization-space-time domain has four dimensions, a four-dimensional search is required. To facilitate observation and analysis, fixed polarization phase angle and intermediate frequency are used to observe the polarizationspace domain pattern, and fixed polarization phase angle and phase difference to observe the space-time domain pattern.

It is assumed that the DOA of the desired signal with $\operatorname{RHCP}$ polarization $\left(\left(\gamma_{0}, \eta_{0}\right)=\left(45^{\circ},-90^{\circ}\right)\right)$ are $20^{\circ}$, and the DOA of the interference signals are $-70^{\circ},-40^{\circ},-20^{\circ}, 20^{\circ}$, $40^{\circ}, 70^{\circ}$. Further, the time of arrival (TOA) of the desired and interference signals is the same. Two scenarios are considered. In scenario 1, the intermediate frequency of the interference signals are $46.52 \mathrm{MHz}$. The parameters are specialized as $\left(\gamma_{i}, \eta_{i}\right)=\left[\left(38^{\circ},-90^{\circ}\right),\left(60^{\circ},-90^{\circ}\right)\right.$,

$\left.\left(45^{\circ},-90^{\circ}\right),\left(18^{\circ},-90^{\circ}\right),\left(40^{\circ},-90^{\circ}\right),\left(85^{\circ},-90^{\circ}\right)\right], 1 \leq i \leq 6$. In scenario 2 , the interference signals are RHCP $\left(\left(\gamma_{i}, \eta_{i}\right)=\left(45^{\circ},-90^{\circ}\right)\right)$ and the intermediate frequencies are $30 \mathrm{MHz}, 35 \mathrm{MHz}, 40 \mathrm{MHz}, 49 \mathrm{MHz}, 55 \mathrm{MHz}$, and 60MHz.

The DPSA-STPAP and APSA-STPAP algorithms are given in Fig. 5 and Fig. 6 for scenario 1, and Fig. 7 and Fig. 8 for scenario 2 . In scenario 1 , when the direction of the interference signal and the desired direction signal are the same, the null can be formed in the polarization domain direction without affecting the reception of the desired signal. And In scenario 2, the null can be formed in the frequency dimension direction without affecting the reception of the desired signal. Therefore, the two algorithms have threedimensional resolution in spatial domain, time domain and polarization domain. When the information of two domains is the same, it will be distinguished and identified from the third domain, so the anti-interference performance is improved.

The results presented show that the proposed algorithm ameliorates the problem with STAP that forms nulls for the interference signals and also attenuates the desired signals when they have the same direction. Polarization information is employed to realize joint polarization-space-time anti-interference. This provides an effective solution for anti-interference when the desired and interference signals have the same DOA.

\subsection{Computational complexity analysis}

Formula (21) gives the solution of the best weight vector in APSA-STPAP algorithm. The solution is mainly for the case of multiple inputs and multiple outputs. For single input and single output, calculating the optimal weight requires the following 4 steps:

(1) The estimated value $\hat{\mathbf{R}}$ of autocorrelation matrix of the received signals is calculated by using $\mathrm{L}$ finite sampling data;

(2) The inverse matrix $\hat{\mathbf{R}}^{-1}$ is solved by $\hat{\mathbf{R}}$;

(3) Calculate the joint steering vector $s_{A S T P}(\theta, f, \gamma, \eta)$

(4) Calculate the product of $\hat{\mathbf{R}}^{-1}$ and $s_{A S T P}(\theta, f, \gamma, \eta)$.

Table 2 and Table 3 give the comparison of the computational complexity of the DPSA-STPAP and APSASTPAP algorithms. It can be seen that the number of real number multiplication, real number division, and real number addition of the APSA-STPAP algorithm has all decreased during the calculation process. It can be seen that the amount of computation is mainly related to the number of snapshots $\mathrm{N}$, the number of array elements $\mathrm{M}$ and the number of timedomain taps K. Fig. 9 shows the comparison curve of the total amount of computation of the two algorithms. The influence of the three variables is observed through the control variables. It can be seen from the figure that the calculation amount of APSA-STPAP algorithm is significantly lower than that of DPSA-STPAP algorithm. It is verified that the space-time-polarization joint anti-interference using APSA 
will reduce the amount of calculation, which is beneficial to engineering realization.

\subsection{Anti-interference performance analysis}

\subsubsection{The influence of the number of time-domain} delay taps anti-interference performance

Based on the simulation conditions, the number of tap and the input SNR are changed. As shown in Fig. 10, the number of taps increases from 1 to 10 , and the influence of the number of taps on the SINR is studied. In the simulation results, three input SNR $(-10 \mathrm{~dB},-20 \mathrm{~dB}$ and $-30 \mathrm{~dB})$ are selected. Every point on the curve is obtained by 500 Monte Carlo experiments.

The simulation results show that under the condition of low SNR, the SNR increases with the increase of tap number, and DPSA-STPAP algorithm is better than APSASTPAP algorithm. In the case of high SNR, the SNR is stable. Before the two taps, DPSA-STPAP algorithm is better than APSA-STPAP algorithm, and after the two taps, the DPSASTPAP algorithm is better than DPSA-STPAP algorithm. This is because with the increase of the number of taps, the notch of zero notch becomes narrow, and the influence on the main lobe is reduced, so that the expected signal loss and the SINR increase. At the same time, when the number of taps increases, the larger the order of the transverse FIR filter, the finer the frequency division, thus the interference signal can be suppressed more accurately. However, the number of taps can not be increased indefinitely, which will increase the computation amount of the algorithm. When the number of taps increases to a certain extent, the SNR remains unchanged, so as to determine the number of tap numbers in time domain.

\subsubsection{The influence of the number of snapshots anti-interference performance}

Based on the simulation conditions, change the number of snapshots and the input SNR. As shown in Fig. 11, the number of taps is increased from 10 to 1000 to study the effect of the number of snapshots on the SINR. In the simulation results, three input SNR of $-10 \mathrm{~dB},-20 \mathrm{~dB}$, and $30 \mathrm{~dB}$ were selected. Each point on the curve is obtained through 500 Monte Carlo experiments. It can be seen from the figure that the SINR increases as the number of snaps increases, and it will remain stable when it increases to a certain level. In the case of small snapshots, the APSASTPAP algorithm is better than the DPSA-STPAP algorithm. As the number of snapshots increases, the output SINR of the DPSA-STPAP algorithm is higher than that of the APSASTPAP algorithm, and the gap between the two algorithms will continue to decrease as the SNR increases.

\subsubsection{The influence of the input SNR on anti- interference performance}

Fig. 12 shows the effect of the input SNR on the output SINR of the two algorithms. The input SNR varies from $40 \mathrm{~dB}$ to $-10 \mathrm{~dB}$. The experimental results are obtained after 500 Monte Carlo experiments are averaged. It can be seen from the figure that the output SINR of the DPSA-STPAP algorithm is higher than that of the APSA-STPAP algorithm, and the gap between the two algorithms will continue to decrease as the SNR increases. The maximum difference between the output SINR of the two algorithms is about 3dB, which shows that through the improvement of the APSA, although the dipole and the degree of freedom of antiinterference are halved, the anti- interference performance has little effect, and the gap is in the acceptable range.

\section{Conclusions and discussion}

This paper implements the APSA-STPAP algorithm based on LCMV criterion, improves the traditional spacetime adaptive algorithm, introduces the polarization domain information, realizes the joint anti-interference of space, time and polarization domain. And It effectively suppresses the interference signal, enhances the reception of the desired signal, and further improves the anti-interference performance. At the same time, compared with DPSA, APSA reduces the computational complexity of the algorithm and is easy to implement in engineering. Simulation results show that APSA-STPAP algorithm can maintain good antiinterference performance when the electric dipole and antiinterference degree of freedom are reduced by half. And it can achieve similar anti-interference effect as DPSA-STPAP algorithm. The output SINR of DPSA-STPAP algorithm is about 3dB higher than APSA-STPAP algorithm. There is little difference between the anti-interference performance of APSA and DPSA. This paper provides a theoretical basis for the application of polarization sensitive array in multidimensional anti-interference, and has a broad application prospect.

Although this paper uses APSA to reduce the amount of computation of multi-dimensional domain joint antiinterference, there are some shortcomings and limitations. Due to the complex manufacturing process and high cost of polarization sensitive array, it brings some difficulties in practical application, so this paper only uses MATLAB simulation experiment to verify the effectiveness and feasibility of the algorithm, without considering the practical application. The research in this paper is carried out in the ideal environment, without considering the influence of error factors in the actual environment, such as the polarization error caused by the inconsistency of the electromagnetic characteristics between the array elements and the coupling effect between the antenna poles, the channel error and the array element position error caused by the antenna installation position deviation.

\section{Abbreviation comparison table}

\begin{tabular}{cc}
\hline Abbreviation & English Name \\
\hline APSA & Alternating Polarization Sensitive Array \\
DPSA & Dual Polarization Sensitive Array \\
LCMV & Linear Variance Minimum Criterion \\
GNSS & Global Navigation Satellite System \\
STAP & Signal Time Adaptive Processing \\
SPTP & Signal Polarization Adaptive Processing \\
STPAP & Space-Time-Polarization Adaptive \\
FIR & Processing \\
\hline
\end{tabular}




\begin{tabular}{cc}
\hline ULA & Uniform Linear Array \\
TE & Transverse Electric \\
RF & Radio Frequency \\
APSA-STPAP & Alternating Polarization Sensitive Array \\
& Space-Time-Polarization Adaptive \\
DPSA-STPAP & Processing \\
& Dual Polarization Sensitive Array \\
Space-Time-Polarization Adaptive & Processing \\
SNR & Signal to Noise Ratio \\
SINR & Signal to Interference and Noise Ratio \\
DOA & Direction of Arrive \\
HP & Horizontal Polarization \\
VP & Vertical Polarization \\
EP & Elliptical Polarization \\
RHCP & Right-Handed Circular Polarization \\
LHCP & Left-Handed Circular Polarization \\
\hline
\end{tabular}

\section{Declarations}

\subsection{Ethics approval and consent to participate}

This paper does not involve the research requiring ethical approval, which is not applicable to this paper.

\subsection{Consent for publication}

My manuscript doesn't contains any individual person's data in any form (including individual details, images or videos), which is not applicable to this paper.

\subsection{Availability of data and material}

Data sharing not applicable to this article as no datasets were generated or analysed during the current study.

\subsection{Competing interests}

The authors declare that this article content has no conflict of interest.

\subsection{Funding}

This work was supported by the National Natural Science Foundation of China (Grant No. 61701462), the Marine S\&T fund of Shandong Province for Pilot National Laboratory for Marine Science and Technology (Qingdao) (Grant No. 2018SDKJ0210), and the Open Studio for Marine High Frequency Communications, Qingdao National Laboratory for Marine Science and Technology.

\subsection{Authors' contributions}

In this paper, a joint space-time-polarization antiinterference method based on the alternating polarization sensitive array is implemented. The method uses the alternating polarization sensitive array as the receiving model, which reduces the computational complexity and mutual coupling effect of the method. Based on the multi linear constraint criterion and space-time adaptive processing, according to the polarization characteristics of desired signals and interference signals, the polarization information of signals is added. Compared with the traditional methods, this method can effectively reduce the influence of cross coupling effect of polarization sensitive array with orthogonal double dipoles, and does not affect the anti-interference performance. It effectively solves the problem that when the direction of the expected satellite signal and the interference signal is close or the interval is small, the satellite signal is attenuated, thus affecting the satellite communication and positioning. It effectively solves the problem that the two-dimensional information is similar The problem of the failure of antiinterference ability. It expands the two-dimensional domain information to three-dimensional domain information to realize the joint anti-interference of space-time-polarization in three-dimensional domain, which provides a theoretical basis for the anti-interference of signals.

\subsection{Acknowledgements}

I would like to express my gratitude to all those who helped me during the writing of this thesis.

I gratefully acknowledge the help of my supervisor, Ms. Lyu Tingting, who has offered me valuable suggestions in the academic studies. In the preparation of the thesis, she has spent much time reading through each draft and provided me with inspiring advice. Without her patient instruction, insightful criticism and expert guidance, the completion of this thesis would not have been possible.

I also owe a special debt of gratitude to Prof. Zhang Hao and Prof. T. Aaron Gulliver, I do appreciate their patience, encouragement, and professional instructions during my thesis writing. I have benefited a lot and academically prepared for the thesis.

I should finally like to express my gratitude to my beloved parents who have always been helping me out of difficulties and supporting without a word of complaint.

\section{References}

[1] Widrow, B., Mantey, P. E., Griffiths, L. J., et al.: 'Adaptive antenna systems', Proc. IEEE, 1967, 55, (12), pp. 2143-2159.

[2] Reed, I. S., Mallett, J. D., Brennan, L. E.: 'Rapid convergence rate in adaptive arrays', IEEE Transactions on Aerospace and Electronic Systems, 1974, 10, (6), pp. 853-863.

[3] Brennan, L. E., Reed, L. S.: 'Theory of adaptive radar', IEEE Transactions on Aerospace and Electronic Systems, 1973, 9, (2), pp. 237-252.

[4] Klemm, R.: 'Introduction to space-time adaptive processing', Electronics \& Communication Engineering Journal, 1999, 11, (1), pp. 5-12.

[5] Richard K.: 'Applications of Space-Time Adaptive Processing' (IET Digital Library, 2004).

[6] Fante, R. L., Vaccaro, J. J.: 'Wideband cancellation of interference in a GPS receive array', IEEE Transactions on Aerospace and Electronic Systems, 2000, 36, (2), pp. $549-564$. 
[7] Chen, F.Q., Nie, J.W., Li, B.Y., et al.: 'Distortionless space-time adaptive processor for global navigation satellite system receiver', Electronics Letters, 2015, 51, (25), pp. 2138-2139.

[8] Compton, R.T.: 'The tripole antenna: An adaptive array with full polarization flexibility', IEEE Transactions on Antennas and Propagation, 1981, 29, (6), pp. 944-952.

[9] Compton, R.T.: 'On the performance of a polarization sensitive adaptive array', IEEE Transactions on Antennas and Propagation, 1981, 29, (5), pp. 718-725.

[10] Cheuk, W. C., Trinkle, M., and Gray, D. A.: 'Nullsteering LMS dual-polarised adaptive antenna arrays for GPS', Positioning, 2005, 1, (9), pp. 258-267.

[11] Wang, J., and Moeness, G. A.: 'Multiple interference cancellation performance for GPS receivers with dualpolarized antenna arrays', EURASIP Journal on Advances in Signal Processing, 2008.

[12] Zhan, Y.H., Li, S.X., Wang, Z.: 'Algorithm and performance analysis of GPS single dual polarized antenna anti interference', Journal of the National University of Defense Technology, 2009, 31, (1), pp. 95-98.

[13] Fohlmeister, F., Iliopoulos, A., Sgammini, M., et al.: 'Dual polarization beamforming algorithm for multipath mitigation in GNSS', Signal Processing, 2017, 138, pp. 86-97.

[14] Fante, R. L., Vaccaro, J. J.: 'Evaluation of adaptive space-time-polarization cancellation of broadband interference', Proc. IEEE Position Location and Navigation Symposium, Palms Springs, CA, Apr. 2002, pp. 1-3.

[15] Zhang, X.F., Wang, F., Xu, D.Z.: 'Theory and application of array signal processing' (National Defense Industry Press, 2013, 1st).

[16] Zhang, X.F., Xu, H.W., Qiu, X.F.: 'Array signal processing and MATLAB implementation' (Electronics Industry Press, 2015, 1st edn.).

[17] Xu, Y.G., Liu, Z.W., Gong, X.F.: 'Polarization sensitive array signal processing' (Beijing Institute of Technology Press, 2013, 1st edn.).

[18] Xu, Z.H., Xiong, Z.Y., Xiao, S.P.: 'A novel alternate polarization array and its filtering performance', IEEE RADAR, 2011, pp. 1890-1892.

[19] Xu, Z.H., Zhang, L., Wu, D. J., et al.: 'Research on filtering performance of alternating polarization array', Journal of the National University of Defense Technology, 2012, 34, (5), pp. 49-54.

[20] Dou, H. J., Xiao, D. L., Zhang, S. F.: 'Performance analysis of alternating polarization sensitive array projection filtering', Journal of Beijing University of Technology, 2017, 2017, (1).

[21] Wang, X. , Li, J., Zhang, M., et al.: 'Space-timepolarization adaptive antenna arrays for GNSS receivers', Journal of Physics Conference Series, 2019, $1169,(1)$.

[22] Wang, H., Yao, Z., Yang, J., et al.: 'A novel beamforming algorithm for GNSS receivers with dualpolarized sensitive arrays in the joint space-timepolarization domain', Sensors, 2018, 18, (12), art. 4506.

[23] Wu, J., Zhu, X.J., He, X.M., Gao, Z.Y., Fang, Y.B.: 'Joint estimation of DOA and polarisation for radar signals based on dual-polarised antenna array', IET Microwaves, Antennas \& Propagation, 2020.
[24] Wang, X. F., Liu, J. Q., Han, C., et al.: 'Navigation antiinterference method based on polarization sensitive array', Missile and Space Carrier Technology, 2017, 2017, (2), pp. 50-52.

[25] Chen, Y.H., et al.: 'Real-time software receiver for GPS controlled reception pattern antenna array processing', Proc. International Technical Meeting of the Satellite Division of the Institute of Navigation, Sep. 2010, pp. 1932-1941.

[26] Elnashar A.: 'Efficient implementation of robust adaptive beamforming based on worst-case performance optimisation', IET Signal Processing, 2008, 2, (2), pp. 1. 
Figures

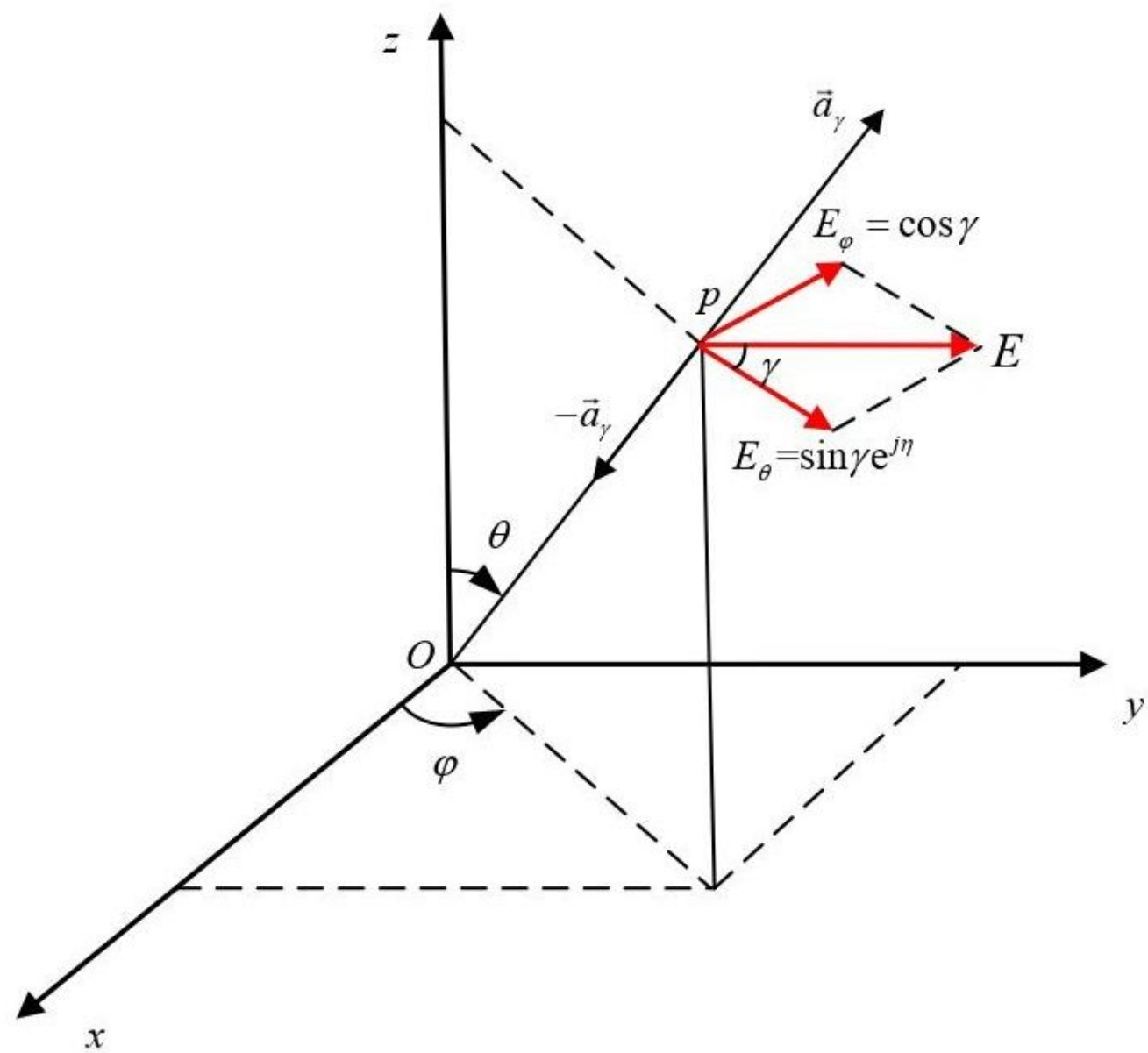

Figure 1

Transverse electric wave 


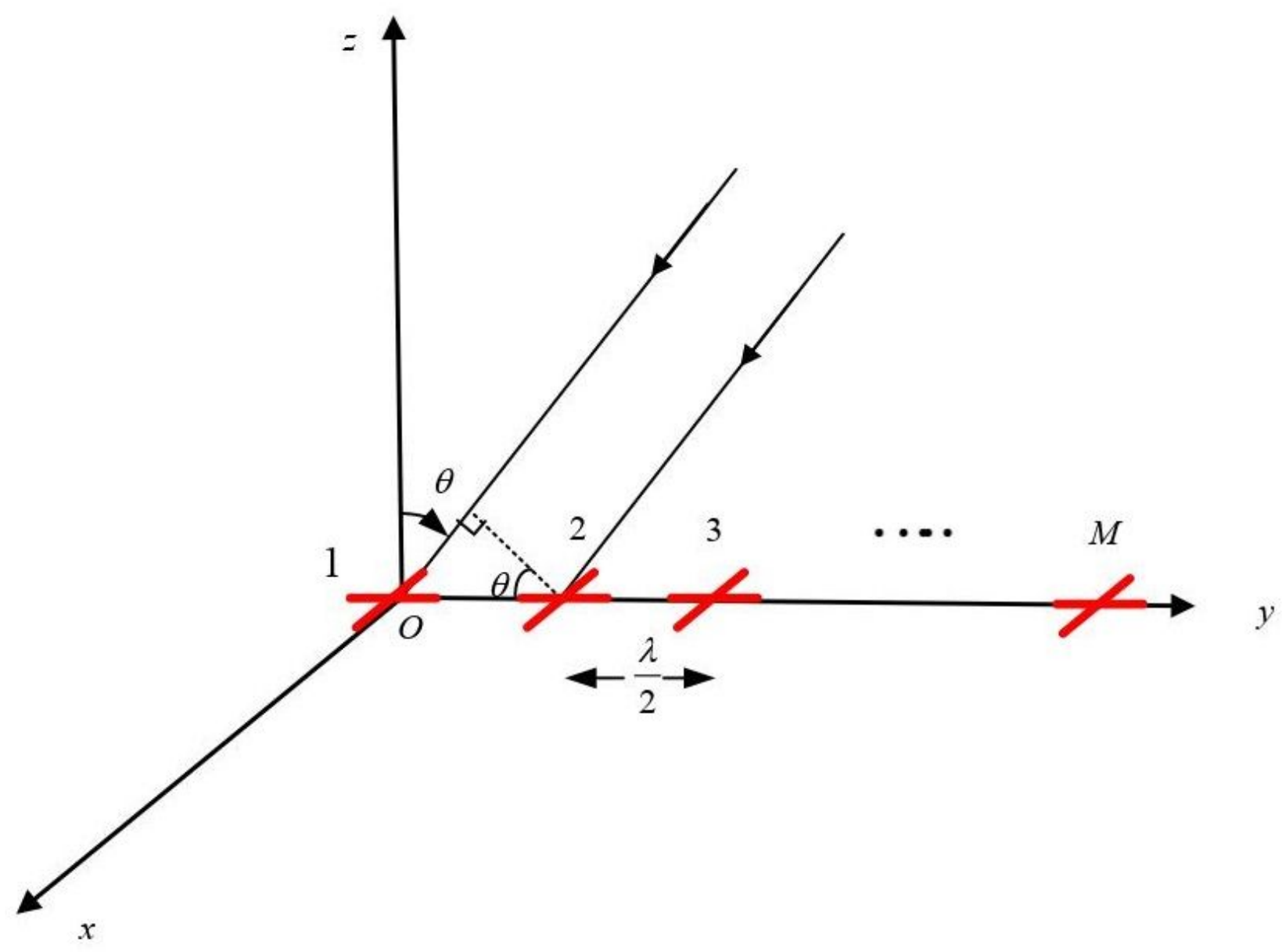

Figure 2

Dual-polarized ULA array 


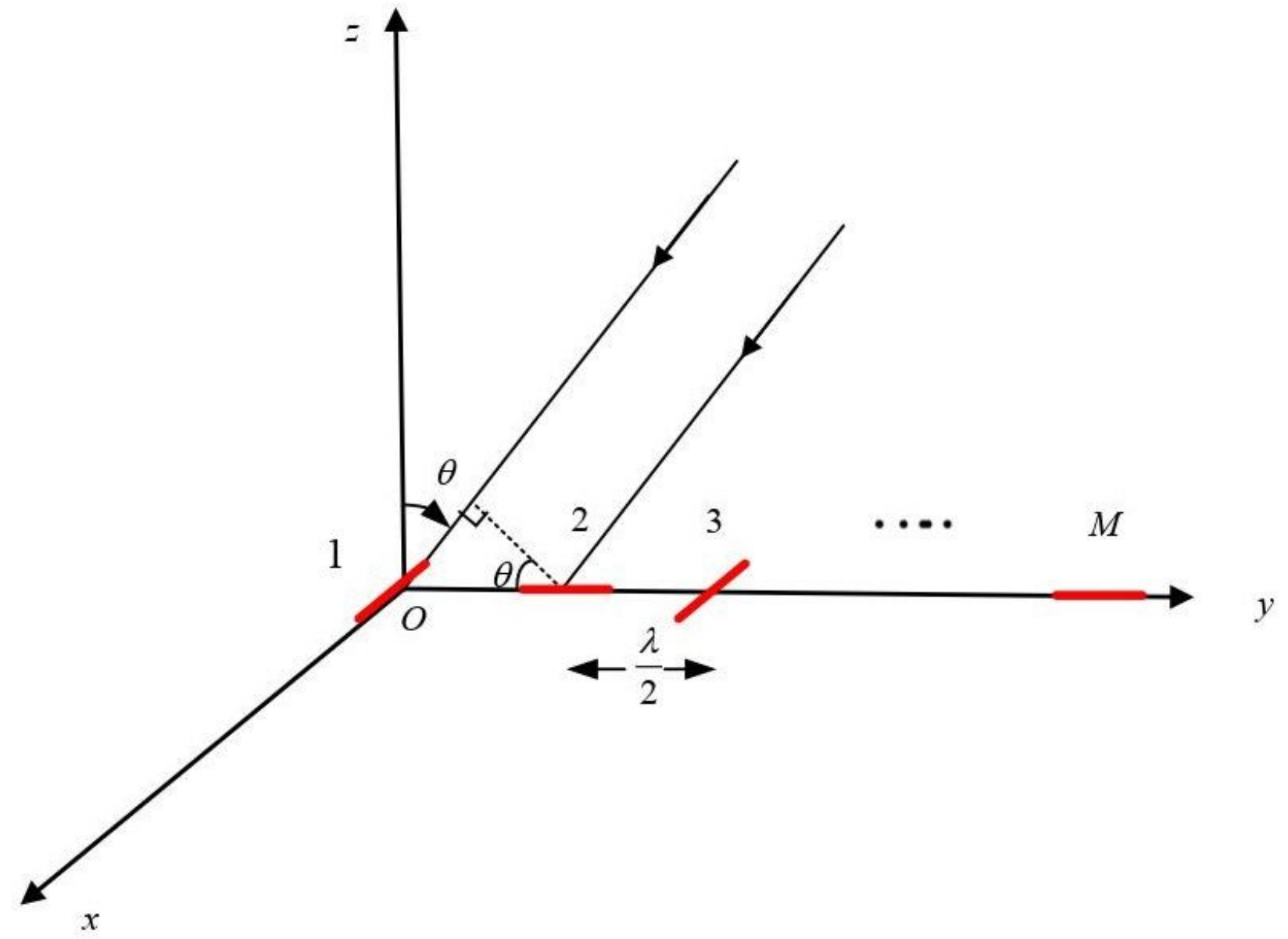

Figure 3

ASPA ULA array 
Element 1

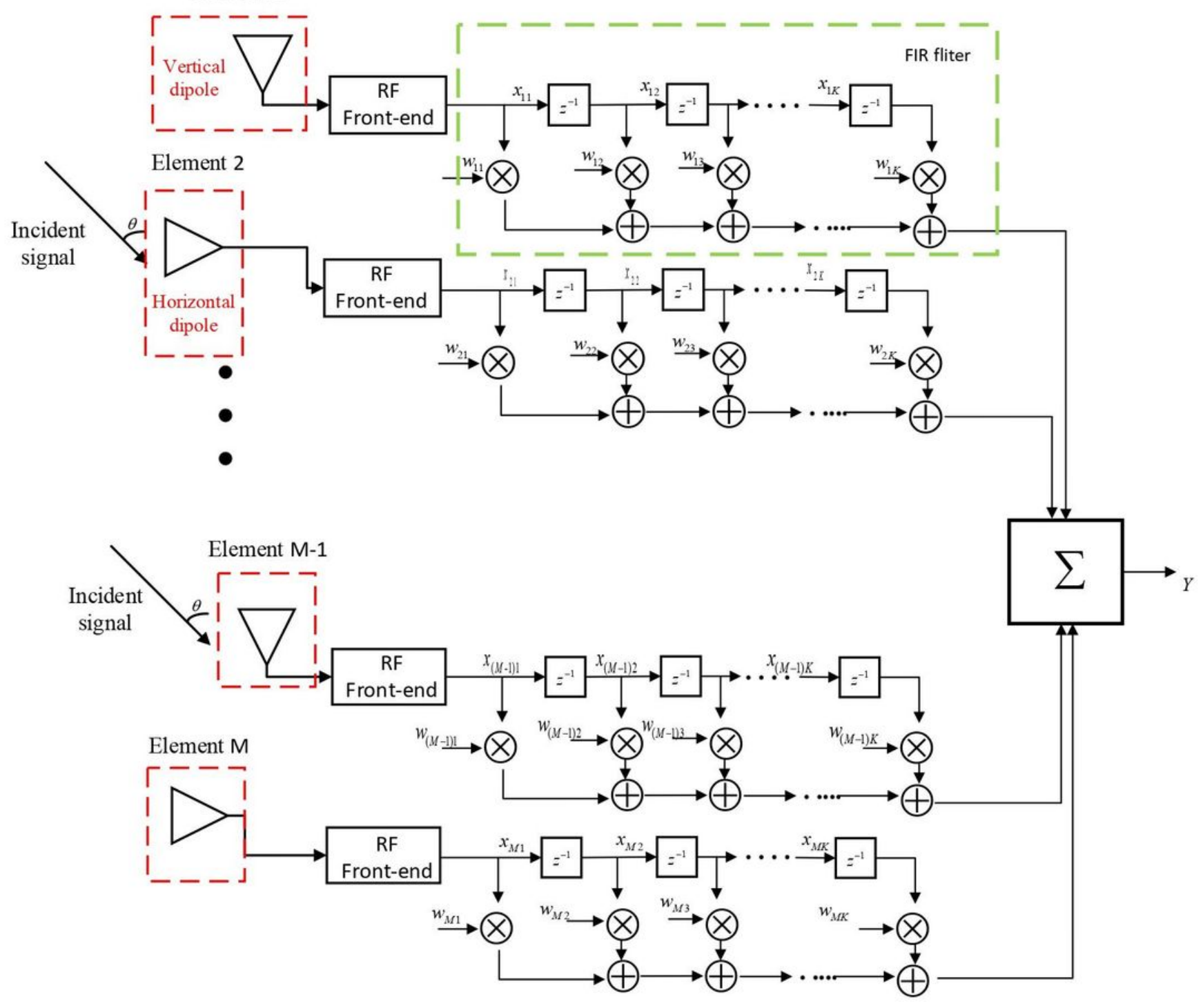

Figure 4

The APSA-STPAP structure 

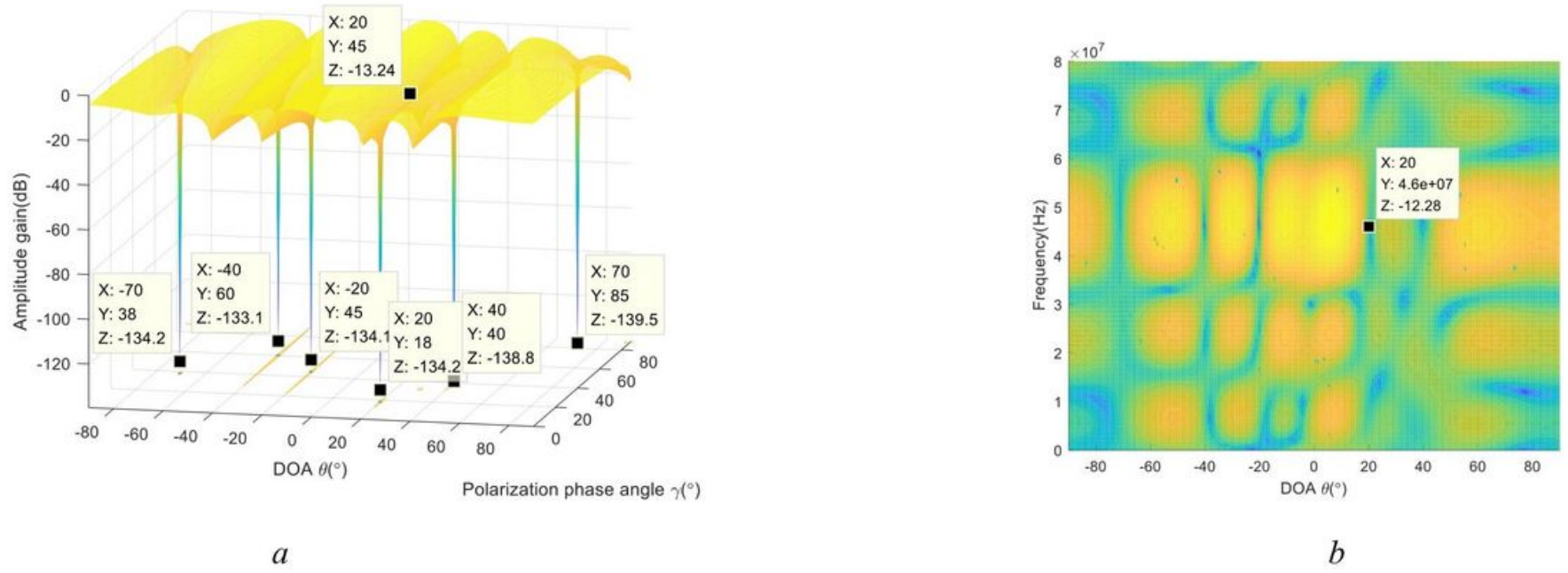

\section{Figure 5}

Three dimensional beam pattern of APSA-STPAP algorithm (a) Space-polarization domain nulling pattern. (b) Top view of space-time domain nulling pattern.
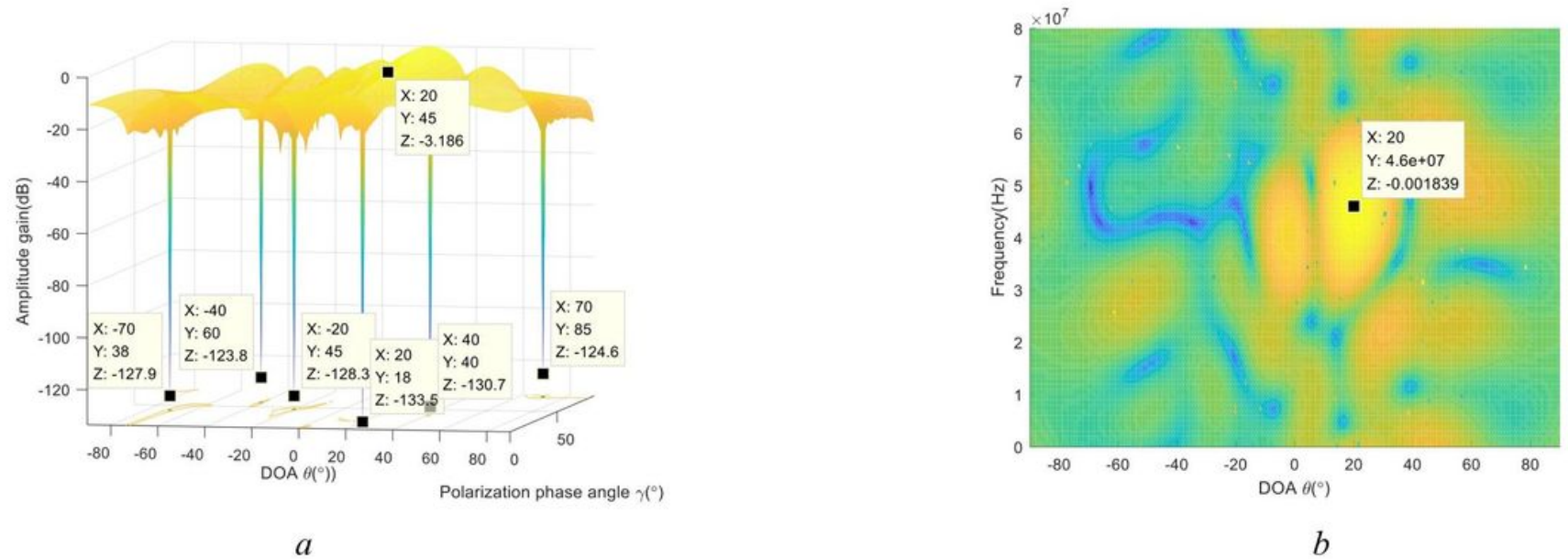

\section{Figure 6}

Three dimensional beam pattern of DPSA-STPAP algorithm (a) Space-polarization domain nulling pattern. (b) Top view of space-time domain nulling pattern. 


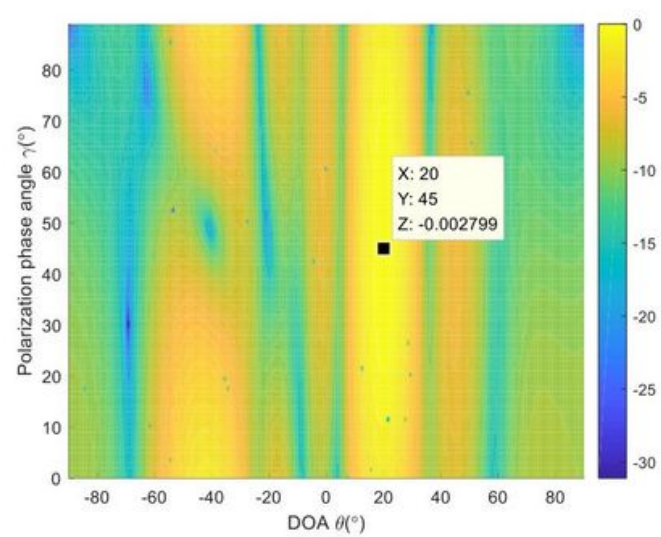

$a$

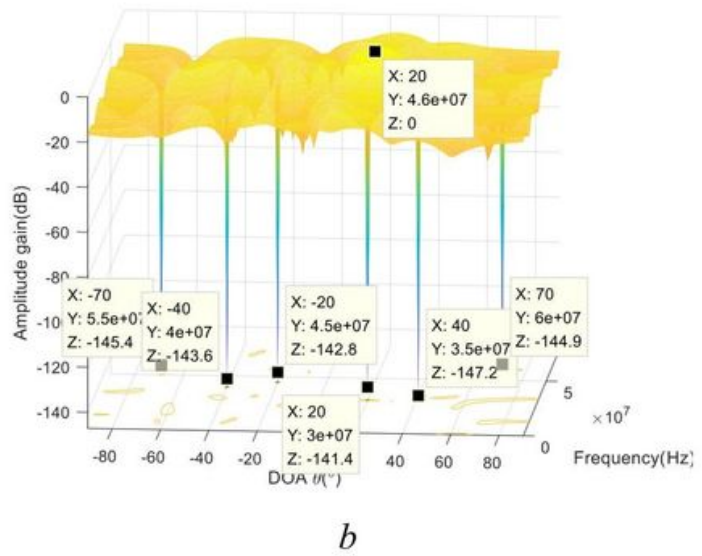

Figure 7

Three dimensional beam pattern of APSA-STPAP algorithm (a) Space-polarization domain nulling pattern. (b) Top view of space-time domain nulling pattern.

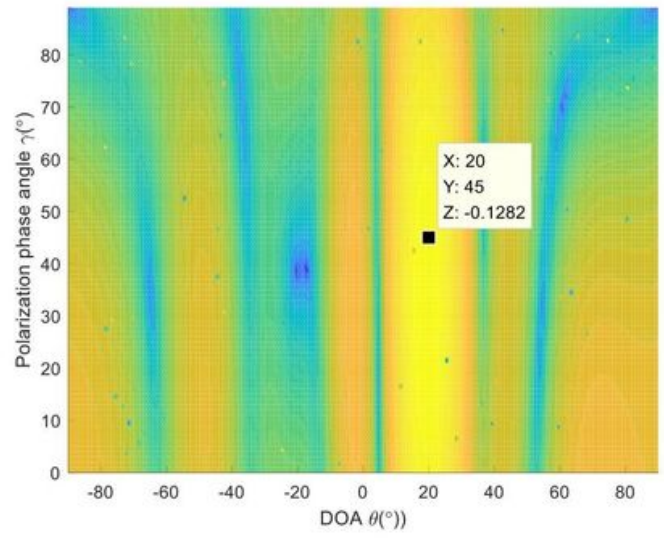

$a$

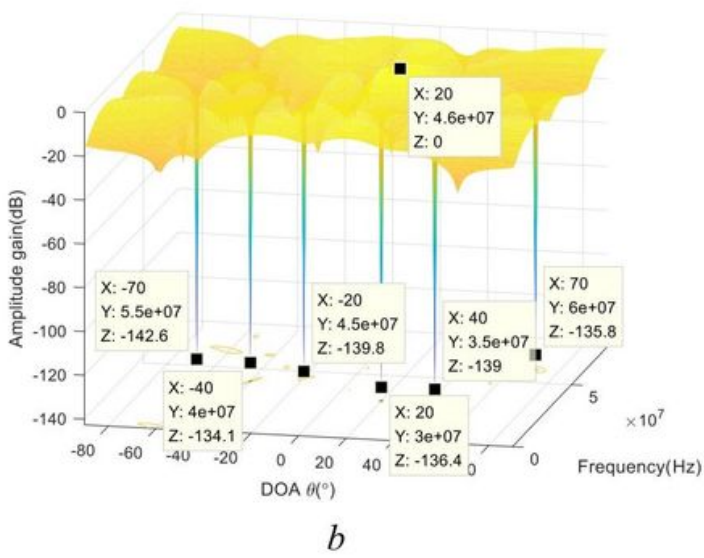

Figure 8

Three dimensional beam pattern of DPSA-STPAP algorithm (a) Space-polarization domain nulling pattern. (b) Top view of space-time domain nulling pattern. 

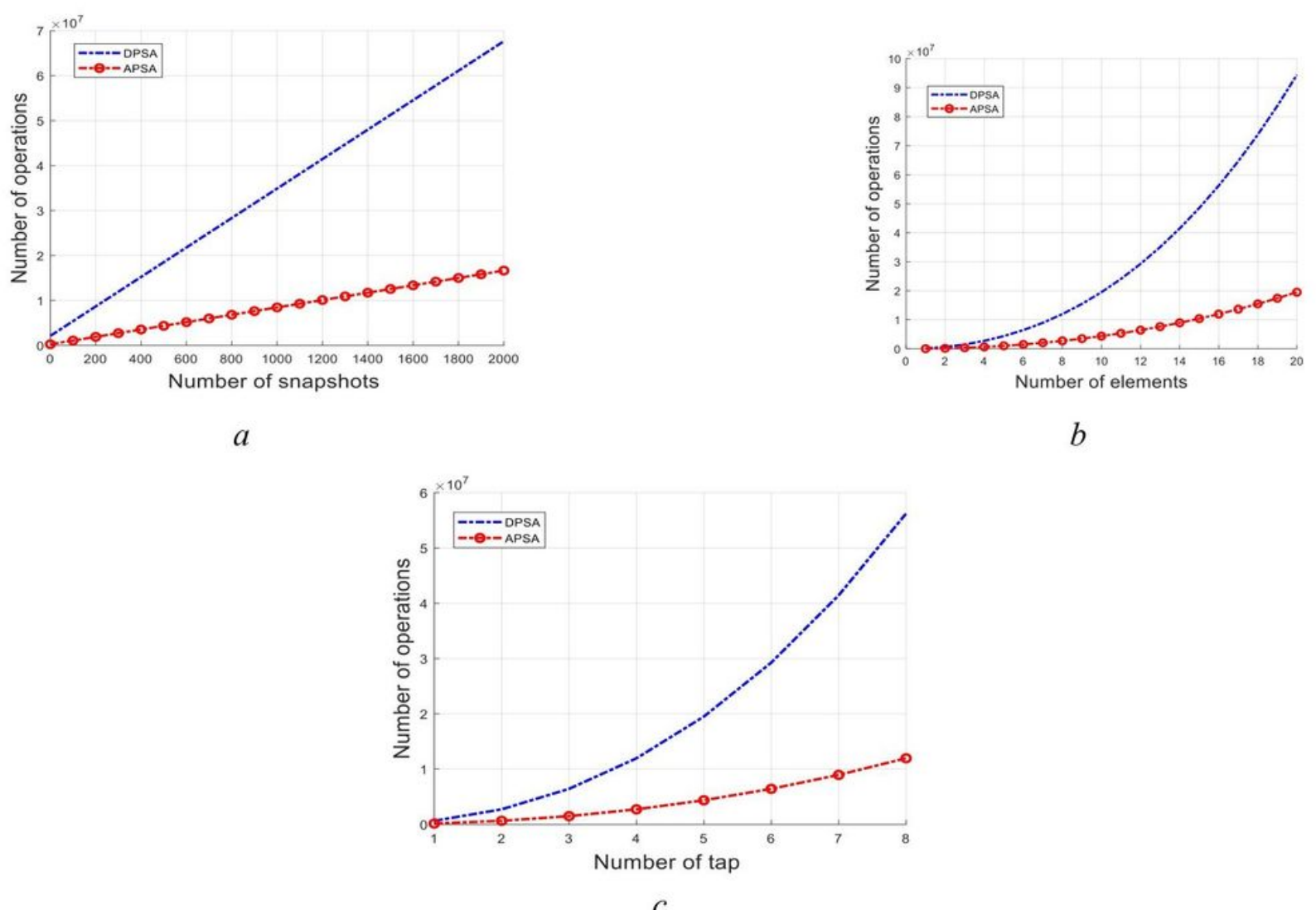

$c$

\section{Figure 9}

Comparison of calculation amount between APSA-STPAP and DPSA-STPAP. (a) The relationship between the number of snapshots and the amount of computation; (b) the relationship between the number of array elements and the amount of computation; (c) the relationship between the number of time taps and the amount of computation. 


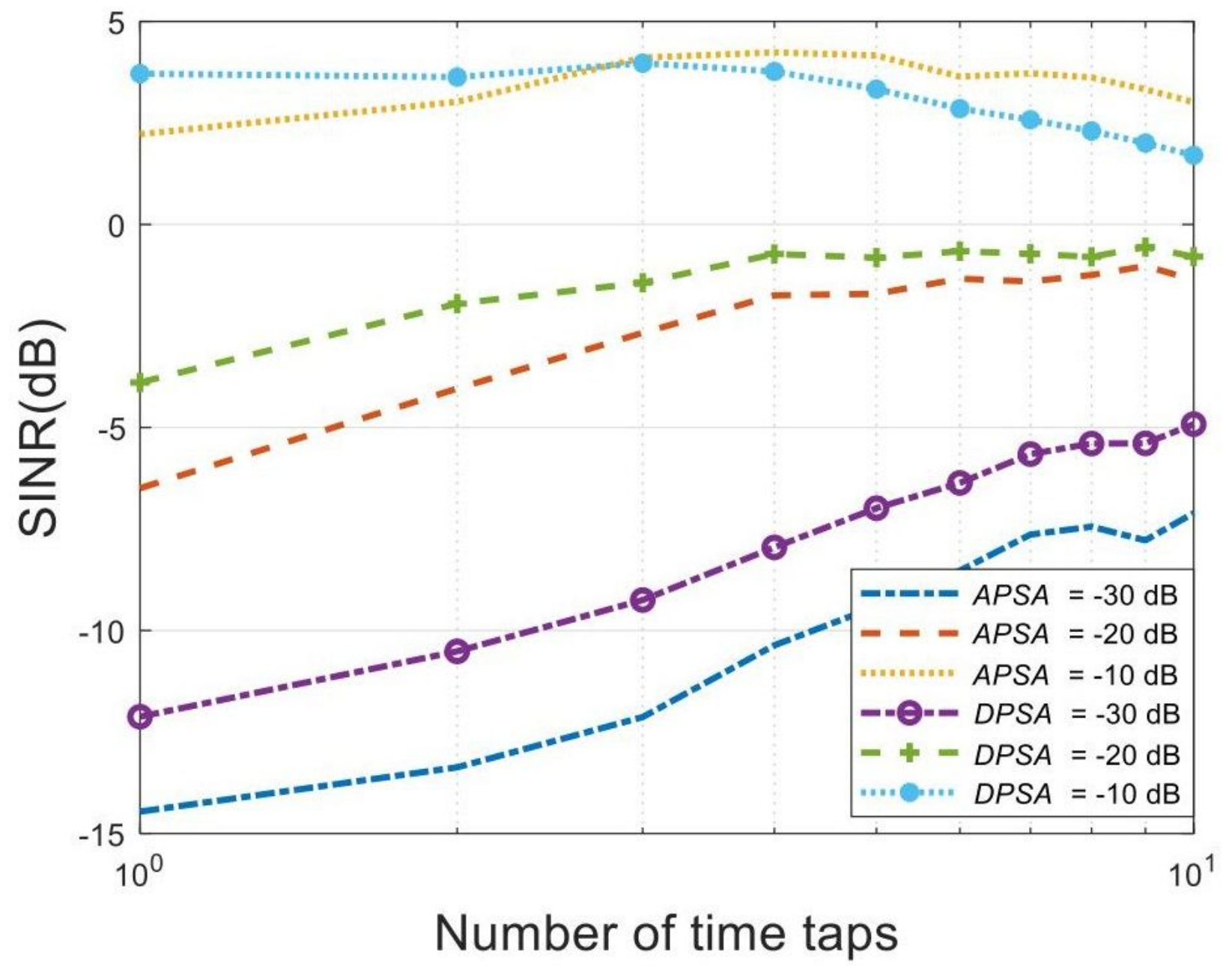

Figure 10

The influence of the number of time-domain delay 


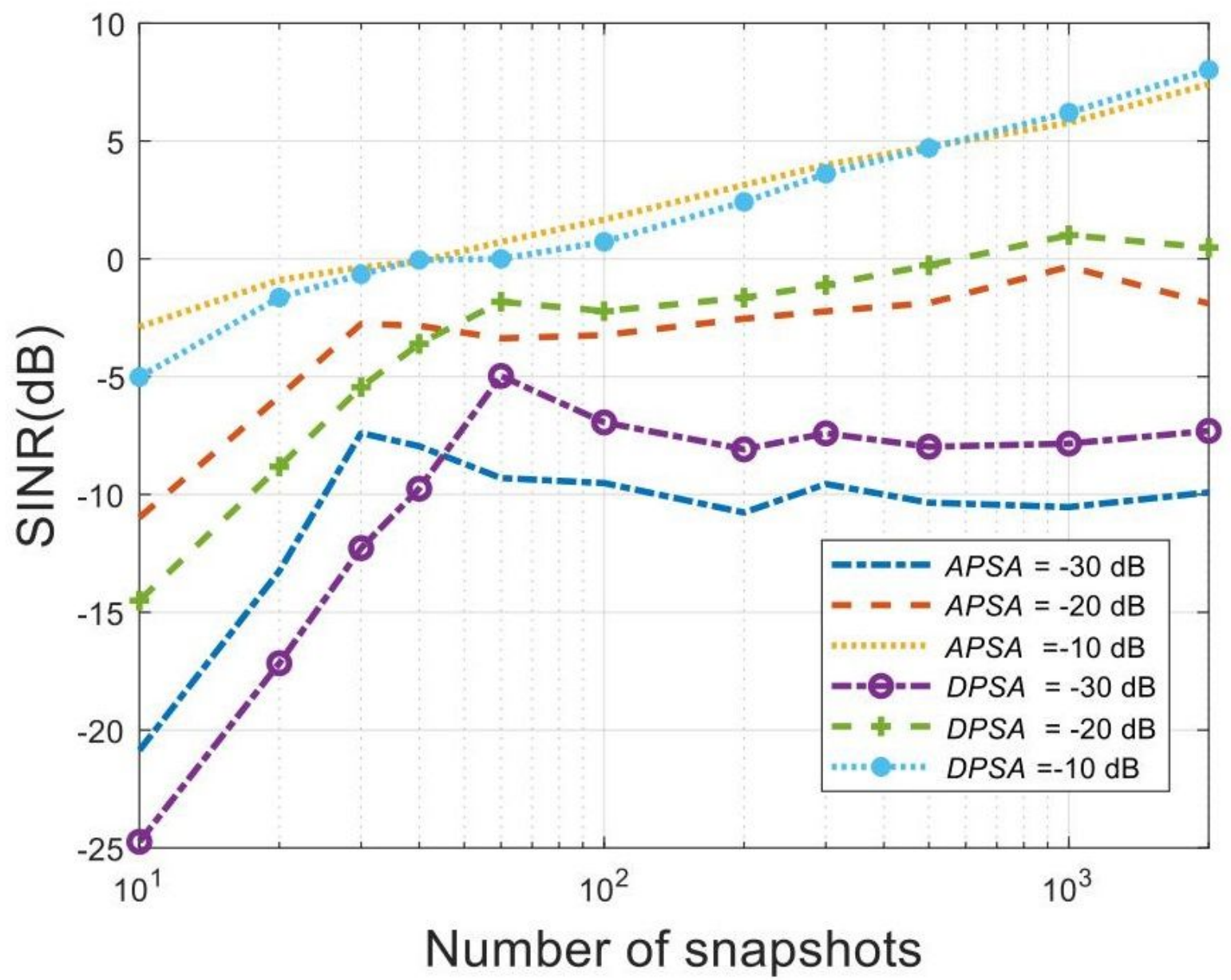

Figure 11

The influence of the number of snapshots anti 


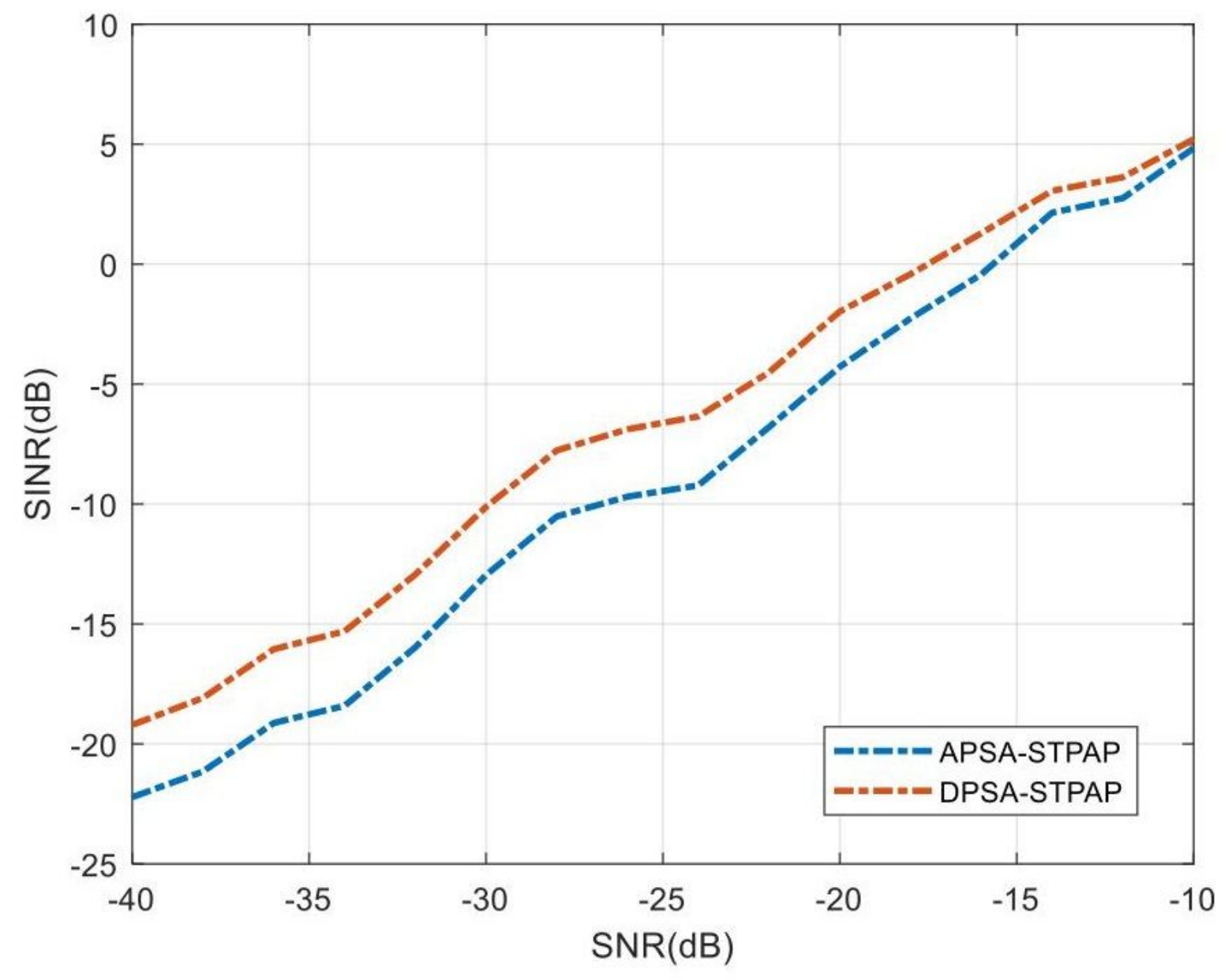

Figure 12

The influence of the input SNR on anti

\section{Supplementary Files}

This is a list of supplementary files associated with this preprint. Click to download.

- Table.pdf 